\begin{tabular}{|c|l|}
\hline Title & Reconstruction of Inclusions for the Inverse Boundary V alue Problem with Mixed Type Boundary Condition \\
\hline Author(s) & Daido, Y.; Ikehata, M.; Nakamura, G. \\
\hline Citation & Hokkaido University Preprint Series in Mathematics, 582, 1-18 \\
\hline Issue Date & 2003-02 \\
\hline DOI & 10.14943/83727 \\
\hline Doc URL & http://hdl.handle.net/2115/69331 \\
\hline Type & bulletin (article) \\
\hline File Information & pre582.pdf \\
\hline
\end{tabular}

Instructions for use 
Reconstruction of Inclusions for the Inverse Boundary Value Problem with Mixed Type Boundary Condition

Yuki Daido, Masaru Ikehata and Gen Nakamura

Series \#582. February 2003 


\section{HOKKAIDO UNIVERSITY PREPRINT SERIES IN MATHEMATICS}

\#556 S. Izumiya, K. Saji and N. Takeuchi, Singularities of line congruences, 17 pages. 2002.

\#557 S. Izumiya, D. Pei and M. C. Romero Fuster, The lightcone Gauss map of a spacelike surface in Minkowski 4-space, 21 pages. 2002.

\#558 S. Izumiya, D. Pei and M. C. Romero Fuster, Umbilicity of spacelike submanifolds of Minkowski space, 14 pages. 2002.

\#559 S. Izumiya, D. Pei and M. Takahashi, Curves and surfaces in Hyperbolic space, 16 pages. 2002.

\#560 J. Kato, On the uniqueness of nondecaying solutions for the Navier-Stokes equations, 19 pages. 2002.

\#561 M. Jinzenji and T. Sasaki, An Approach to $\mathcal{N}=4 A D E$ gauge Theory on $K 3,29$ pages. 2002.

\#562 T. Nakazi and T. Yamamoto, Norms of some singular integral operators on weighted $L^{2}$ spaces, 27 pages. 2002.

\#563 A. Harris and Y. Tonegawa, A $\bar{\partial} \partial$-poincaré lemma for forms near an isolated complex singularity, 8 pages. 2002.

\#564 M. Takahashi, Bifurcations of ordinary differential equations of Clairaut type, 23 pages. 2002.

\#565 G. Ishikawa, Classifying singular Legendre curves by contactomorphisms, 17 pages. 2002.

\#566 G. Ishikawa, Perturbations of Caustics and fronts, 17 pages. 2002.

\#567 Y. Giga and O. Sawada, On regularizing-decay rate estmates for solutions to the Navier-Stokes initial value problem, 12 pages. 2002.

\#568 T. Miyao, Strongry supercommuting serf-adjoint operators, 34 pages. 2002.

\#569 Jun-Muk Hwang and K. Yamaguchi, Characterization of Hermitian symmetric spaces by fundamental forms, 10 pages. 2002.

\#570 H. Ishii and T. Mikami, Convexified Gauss curvature flow of bounded open sets in an anisotropic external field: a stochastic apporoximation and PDE, 37 pages. 2002.

\#571 Y. Nakano, Minimization of shortfall risk in a jump-diffusion model, 10 pages. 2002.

\#572 K. Izuchi and T. Nakazi, Backward Shift Invariant Subspaces in the Bidisc, 8 pages. 2002.

\#573 S. Izumiya, D. Pei and M. C. Romero-Fuster, The horospherical geometry of surfaces in Hyperbolic 4-space, 17 pages. 2002.

\#574 S. Izumiya and M. C. Romero-Fuster, The hyperbolic Gauss-Bonnet type theorem, 10 pages. 2002.

\#575 S. Izumiya and S. Janeczko, A symplectic framework for multiplane gravitational lensing, 19 pages. 2002.

\#576 S. Izumiya, M. Kossowski, D. Pei and M. C. Romero-Fuster, Singularities of $C^{\infty}$-lightlike hypersurfaces in Minkowski 4-space, 18 pages. 2002.

\#577 S. Izumiya, D. Pei and M.Takahashi, Evolutes of hypersurfaces in Hyperbolic space, 21 pages. 2002.

\#578 Y. Giga, S. Matsui and S. Sasayama, Blow up rate for semilinear heat equation with subcritical nonlinearity, 29 pages. 2002.

\#579 M. Tsujii, Physical measures for partially hyperbolic surface endomorphisms, 71 pages. 2003.

\#580 Y. Giga and K. Yamada, On viscous Burgers-like equations with linearly growing initial data, 19 pages. 2003.

\#581 T. Nakazi and T. Osawa, Spectra Of Toeplitz Operators And Uniform Algebras, 9 pages. 2003. 


\title{
Reconstruction of Inclusions for the Inverse Boundary Value Problem with Mixed Type Boundary Condition
}

\author{
YUKI DAIDO $^{a}$, MASARU IKEHATA ${ }^{b}$ and GEN NAKAMURA ${ }^{a}$
}

${ }^{a}$ Department of Mathematics, Hokkaido University, Sapporo 060-0810, Japan; ${ }^{b}$ Department of Mathematics, Faculty of Engineering Gunma University, Kiryu 376-8515, Japan

We consider an inverse boundary value problem for identifying the inclusion inside a known anisotropic conductive medium. We give a reconstruction procedure for identifying the inclusion from the Dirichlet-Neumann map or the Neumann-Dirichlet map associated with the mixed type boundary condition.

Keywords: Inverse boundary value problem; Conductiviy equation; Probe method; Mixed type boundary condition

AMS Classifications: 35J25; 35Q72; 35R30

\section{INTRODUCTION}

Let $\Omega \subset \boldsymbol{R}^{n}$ ( $n=2$ or 3 ) be a bounded domain with $C^{2}$ boundary $\Gamma$. $\Omega$ is considered as a conductive medium with conductivity:

$$
\gamma=\gamma_{0}+\chi_{D} h \gamma_{0}
$$

with a matrix $\gamma_{0}=\left(\gamma_{0 i j}\right) \in C^{0,1}(\bar{\Omega})$ and a real valued scalar function $h \in L^{\infty}(\Omega)$. Here $D$ is a bounded domain with Lipschitz boundary $\partial D$ such that $\bar{D} \subset \Omega, \Omega \backslash \bar{D}$ is connected, $\chi_{D}$ is the characteristic function of $D$ and $C^{0,1}(\bar{\Omega})$ is the space of functions which are Lipschitz continuous on $\bar{\Omega}$. We assume that $\gamma_{0}=\left(\gamma_{0 i j}(x)\right)$ is a symmetric matrix satisfying

$$
\sum_{i, j=1}^{n} \gamma_{0 i j}(x) \xi_{i} \xi_{j} \geq C_{1}|\xi|^{2} \quad\left(\xi=\left(\xi_{1}, \cdots, \xi_{n}\right) \in R^{n} \text {, a.e. } x \in \bar{\Omega}\right)
$$

for some constant $C_{1}>0$, and for any $a \in \partial D$, there exists a $\delta>0$ such that either

$$
h(x) \geq C_{2} \quad\left(\text { a.e. } x \in B_{\delta}(a)\right)
$$

or

$$
h(x) \leq-C_{2} \quad\left(\text { a.e. } x \in B_{\delta}(a)\right)
$$


holds for some constant $C_{2}>0$, where $B_{\delta}(a):=\left\{x \in \boldsymbol{R}^{n} ;|x-a|<\delta\right\}$. Moreover, we assume that $h \in C^{0, \alpha}$ near $\partial D$ with $0<\alpha<1$ for $n=2$ and $\frac{1}{2}<\alpha<1$ for $n=3$.

Let $\Gamma$ consist of two parts. That is

$$
\Gamma=\overline{\Gamma_{D}} \cup \overline{\Gamma_{N}}
$$

where $\Gamma_{D}, \Gamma_{N}$ are open subsets of $\Gamma$ such that $\Gamma_{D} \cap \Gamma_{N}=\phi, \Gamma_{D} \neq \phi, \Gamma_{N} \neq \phi$ and for $n=3$, the boundaries $\partial \Gamma_{D}, \partial \Gamma_{N}$ of $\Gamma_{D}, \Gamma_{N}$ are $C^{2}$, respectively.

Consider the mixed type boundary value problem:

$$
\left\{\begin{array}{l}
\left(L_{\gamma} u\right)(x):=\sum_{i, j=1}^{n} \partial_{i}\left(\gamma_{i j}(x) \partial_{j} u(x)\right)=F(x) \text { in } \Omega \\
u=f \text { on } \Gamma_{D}, \quad \partial_{L_{\gamma}} u=g \text { on } \Gamma_{N}
\end{array}\right.
$$

for given $f \in \bar{H}^{\frac{1}{2}}\left(\Gamma_{D}\right), g \in \bar{H}^{-\frac{1}{2}}\left(\Gamma_{N}\right), F \in L^{2}(\Omega)$ where $x=\left(x_{1}, \cdots, x_{n}\right), \partial_{i}:=\partial / \partial x_{i}$ and

$$
\left(\partial_{L_{\gamma}} u\right)(x):=\sum_{i, j=1}^{n} \nu_{i} \gamma_{i j}(x) \partial_{j} u(x)
$$

with the unit outer normal vector $\nu=\left(\nu_{1} \cdots \nu_{n}\right)$ of $\Gamma$. Here we have used the notations given in [2] to denote Sobolev spaces.

By Appendix A, there exists a unique solution $u=u(f, g, F) \in \bar{H}^{1}(\Omega)$ to (1.6) with the estimate:

$$
\|u\|_{\bar{H}^{1}(\Omega)} \leq C\left(\|f\|_{\bar{H}^{\frac{1}{2}}\left(\Gamma_{D}\right)}+\|g\|_{\bar{H}^{-\frac{1}{2}}\left(\Gamma_{N}\right)}+\|F\|_{L^{2}(\Omega)}\right),
$$

where the constant $C>0$ does not depend on $f, g, F$.

Moreover, even for $F \in W^{*}$ with $W:=\left\{w \in \bar{H}^{1}(\Omega) ; w=0\right.$ on $\left.\Gamma_{D}\right\}$ and $\operatorname{supp} F \subset \Omega$, we have the similar result that $\|F\|_{L^{2}(\Omega)}$ in (1.8) has to be replaced by $\|F\|_{W^{*}}$. Hereafter, the norm $\|\cdot\|_{W}$ and inner product $($,$) of W$ are those of $\bar{H}^{1}(\Omega)$, and the norm of the dual space $W^{*}$ of $W$ is denoted by $\|\cdot\|_{W^{*}}$.

Next, we define the Dirichlet to Neumann map $\Lambda_{\gamma}$ and the Neumann to Dirichlet map $\Pi_{\gamma}$ as follows.

DEFINITION 1.1 Let $u(f, g, F)$ be the solution to (1.6).

(i) Fixing $g$ and $F$, define $\Lambda_{\gamma}: \bar{H}^{\frac{1}{2}}\left(\Gamma_{D}\right) \rightarrow \bar{H}^{-\frac{1}{2}}\left(\Gamma_{D}\right)$ by

$$
\Lambda_{\gamma} f:=\partial_{L_{\gamma}} u(f, g, F) \text { on } \Gamma_{D}
$$

(ii) Fixing $f$ and $F$, define $\Pi_{\gamma}: \bar{H}^{-\frac{1}{2}}\left(\Gamma_{N}\right) \rightarrow \bar{H}^{\frac{1}{2}}\left(\Gamma_{N}\right)$ by

$$
\Pi_{\gamma} g:=u(f, g, F) \text { on } \Gamma_{N} .
$$


REMARK 1.2 The trace of $\partial_{L_{\gamma}} u(f, g, F) \in \bar{H}^{-\frac{1}{2}}\left(\Gamma_{D}\right)$ exists, because $F \in L^{2}(\Omega)$ or $F \in W^{*}$ with $\operatorname{supp} F \subset \Omega$.

Now, we consider two kinds of inverse problems (IP1) and (IP2):

(IP1) Suppose $\gamma_{0}$ is known and $h, D$ are unknown. Reconstruct $D$ from $\Lambda_{\gamma}$.

(IP2) Suppose $\gamma_{0}$ is known and $h, D$ are unknown. Reconstruct $D$ from $\Pi_{\gamma}$.

THEOREM 1.3 There are reconstruction procedures for the both inverse problems (IP1) and (IP2).

\section{REMARK 1.4}

If it is possible to relate $\Lambda_{\gamma}$ or $\Pi_{\gamma}$ to a Dirichlet to Neumann map $\Lambda_{1, \gamma}$ defined on the boundary of a subdomain $\Omega_{1}$ of $\Omega$ such that $\overline{\Omega_{1}} \subset \Omega, \Omega \backslash \overline{\Omega_{1}}$ is connected and its boundary $\partial \Omega_{1}$ is Lipschitz smooth, then by a similar argument given in [3], $D$ can be reconstructed from $\Lambda_{1, \gamma}$. However, we are interested in obtaining a reconstruction procedure which directly uses $\Lambda_{\gamma}$ or $\Pi_{\gamma}$. For our reconstruction we need to analyze the behavior of the terms with $f, g, F$ in the estimates of indicator functions given in (3.8) and (3.9). We have used the De Giorgi-Nash-Moser theorem for doing this.

The probe method for the inverse boundary value problem with mixed type boundary condition was shown in [4] for identifying cracks. A new ingredient of this paper is an application of the De Giorgi-Nash-Moser theorem (see Appendix B) for estimating the indicator function given later when the conductivity of the inclusion is Hölder continuous near boundary. We also have to point out that the inverse boundary value problem identifying inclusions was initiated by Isakov [6] and there are closely related works done by Potthast and his collaborators ([7]) which use singular solutions for reconstructing an unknown scatter. Our reconstruction procedure without using any reduction to a known reconstruction procedure is totally new.

\section{RECONSTRUCTION PROCEDURE}

DEFINITION 2.1 (needle) We call a nonselfintersecting piecewise $C^{1}$ curve $\mathcal{C}:=\{c(t) ; 0 \leq t \leq 1\}$ joining $c(0), c(1) \in \Gamma$ needle if it satisfies $\mathcal{C} \backslash\{c(0), c(1)\} \subset \Omega$. 


\section{DEFINITION 2.2 (singular solution)}

(i) Fix $x^{0} \in \Omega$ and let $G\left(x-x^{0}\right) \in \mathcal{D}^{\prime}\left(\boldsymbol{R}^{n}\right)$ be a fundamental solution of

$$
\nabla \cdot\left(\gamma_{0}\left(x^{0}\right) \nabla G\left(x-x^{0}\right)\right)+\delta\left(x-x^{0}\right)=0 \text { in } \boldsymbol{R}^{n} .
$$

(ii) Let $H_{j}\left(x, x^{0}\right) \in \mathcal{D}^{\prime}\left(\boldsymbol{R}_{x}^{n}\right)(j=1,2)$ be solutions of

$$
L_{\gamma_{0}} H_{j}\left(x, x^{0}\right)+\delta\left(x-x^{0}\right)=0 \text { in } \Omega
$$

such that

$$
H_{j}\left(x, x^{0}\right)-G\left(x-x^{0}\right) \in \bar{H}^{1}(\Omega)
$$

and

$$
\left\{\begin{array}{l}
\partial_{L_{\gamma_{0}}} H_{1}\left(x, x^{0}\right)=0 \text { on } \Gamma_{N} \\
H_{2}\left(x, x^{0}\right)=0 \text { on } \Gamma_{D} .
\end{array}\right.
$$

We call each $H_{j}\left(x, x^{0}\right)$ singular solution.

REMARK 2.3 The construction of singular solution can be done similarly to Lemma 3 in [5]

Let $\mathcal{C}:=\{c(t) ; 0 \leq t \leq 1\}$ be a needle. By the Runge approximation theorem given in Appendix C, there exist sequences of approximate functions $\left\{v_{1 k}\right\},\left\{v_{2 k}\right\} \subset \bar{H}^{1}(\Omega)$ such that $v_{j k} \rightarrow v_{j}^{\prime}+H_{j}(\cdot, c(t))(k \rightarrow \infty) \quad$ in $\bar{H}_{\text {loc }}^{1}\left(\Omega \backslash \mathcal{C}_{t}\right)$ for each $j(j=1,2)$,

$$
\left\{\begin{array}{l}
L_{\gamma_{0}} v_{1 k}=F \text { in } \Omega \\
\partial_{L_{\gamma_{0}}} v_{1 k}=g \text { on } \Gamma_{N}, \quad \operatorname{supp}\left(\left.v_{1 k}\right|_{\Gamma_{N}}\right) \subset \Gamma_{D}
\end{array}\right.
$$

and

$$
\left\{\begin{array}{l}
L_{\gamma_{0}} v_{2 k}=F \text { in } \Omega \\
v_{2 k}=f \text { on } \Gamma_{D}
\end{array}\right.
$$

where $\mathcal{C}_{t}:=\{c(s) ; 0 \leq s \leq t\}$ and $v_{j}^{\prime} \in \bar{H}^{1}(\Omega)(j=1,2)$ are the solutions to

$$
\left\{\begin{array}{l}
L_{\gamma_{0}} v_{1}^{\prime}=F \text { in } \Omega \\
v_{1}^{\prime}=0 \text { on } \Gamma_{D}, \quad \partial_{L_{\gamma_{0}}} v_{1}^{\prime}=g \text { on } \Gamma_{N}
\end{array}\right.
$$

and

$$
\left\{\begin{array}{l}
L_{\gamma_{0}} v_{2}^{\prime}=F \text { in } \Omega \\
v_{2}^{\prime}=f \text { on } \Gamma_{D}, \quad \partial_{L_{\gamma_{0}}} v_{2}^{\prime}=0 \text { on } \Gamma_{N}
\end{array}\right.
$$

(see Appendix $\mathrm{C}$ for the details). 
DEFINITION 2.4 (indicator function) Let $\mathcal{C}=\{c(t) ; 0 \leq t \leq 1\}$ be a needle, $t(0<t<1)$ satisfy $\mathcal{C}_{t} \cap \bar{D}=\phi$ and $\left\{v_{j k}\right\} \subset \bar{H}^{1}(\Omega)(j=1,2)$ be the sequences of approximate functions given above. Then, we define two indicator functions $I_{1}(t, \mathcal{C})$ and $I_{2}(t, \mathcal{C})$ associated with (IP1) and (IP2):

$$
I_{1}(t, \mathcal{C}):=\lim _{k \rightarrow \infty}\left\langle\left(\Lambda_{\gamma}-\Lambda_{\gamma_{0}}\right)\left(\left.v_{1 k}\right|_{\Gamma_{D}}\right),\left.v_{1 k}\right|_{\Gamma_{D}}\right\rangle_{1}
$$

and

$$
I_{2}(t, \mathcal{C}):=\lim _{k \rightarrow \infty}\left\langle\left.\left(\partial_{L_{\gamma}} v_{2 k}\right)\right|_{\Gamma_{N}},\left(\Pi_{\gamma}-\Pi_{\gamma_{0}}\right)\left(\left.\left(\partial_{L_{\gamma}} v_{2 k}\right)\right|_{\Gamma_{N}}\right)\right\rangle_{2}
$$

where $\langle,\rangle_{1}$ and $\langle,\rangle_{2}$ are the pairings for $\left\{\dot{H}^{-\frac{1}{2}}\left(\overline{\Gamma_{D}}\right), \bar{H}^{\frac{1}{2}}\left(\Gamma_{D}\right)\right\}$ and $\left\{\bar{H}^{-\frac{1}{2}}\left(\Gamma_{N}\right), \dot{H}^{\frac{1}{2}}\left(\overline{\Gamma_{N}}\right)\right\}$, respectively.

DEFINITION 2.5 (first hitting time) Let $\mathcal{C}=\{c(t) ; 0 \leq t \leq 1\}$ be a needle such that $\mathcal{C} \cap \bar{D} \neq \phi$. We define $T(\mathcal{C}, D)$ by

$$
T(\mathcal{C}, D):=\sup \{t ; 0<t<1, c(s) \notin \bar{D}(0 \leq s<t)\} .
$$

We call $T(\mathcal{C}, D)$ the first hitting time of $\mathcal{C}$ to $D$.

DEFINITION 2.6 (detecting time) Let $\mathcal{C}$ be as in Definition 2.5. For the indicator functions $I_{j}(t, \mathcal{C})(j=1,2)$, we define their detecting times $t_{j}(t, \mathcal{C})(j=1,2)$ by

$$
t_{j}(\mathcal{C}, D):=\sup \left\{0<t<1 ; \sup _{0<s<t}\left|I_{j}(s, \mathcal{C})\right|<\infty\right\} .
$$

Then, we have our main theorem

THEOREM 2.7 For each $j(j=1,2)$, we have

$$
T(\mathcal{C}, D)=t_{j}(\mathcal{C}, D) \quad \text { if } \mathcal{C} \cap \bar{D} \neq \phi
$$

Since we can reconstruct $D$ by knowing $t_{j}(\mathcal{C}, D)$ for all possible $\mathcal{C}$, Theorem 2.7 implies Theorem 1.3. 


\section{ESTIMATES OF INDICATOR FUNCTIONS}

In this Section we give some estimates for the indicator functions $I_{j}(t, \mathcal{C})(j=1,2)$. Let $u_{j k} \in \bar{H}^{1}(\Omega)(j=1,2 ; k \in N)$ be

$$
\left\{\begin{array}{l}
u_{1 k}:=u\left(\left.v_{1 k}\right|_{\Gamma_{D}}, g, F\right) \\
u_{2 k}:=u\left(f,\left.\left(\partial_{L_{\gamma_{0}}} v_{2 k}\right)\right|_{\Gamma_{N}}, F\right),
\end{array}\right.
$$

where $u=u(f, g, F)$ is the solution to (1.6). Also, let

$$
w_{j k}:=u_{j k}-v_{j k} \quad(j=1,2 ; k \in \boldsymbol{N}) .
$$

Then, we have

$$
\left\{\begin{array}{l}
L_{\gamma} w_{j k}=\nabla \cdot\left(\gamma_{0}-\gamma\right) \nabla v_{j k} \text { in } \Omega \\
w_{j k}=0 \text { on } \Gamma_{D}, \partial_{L_{\gamma}} w_{j k}=0 \text { on } \Gamma_{N} .
\end{array}\right.
$$

More precisely, $w_{j k} \in W$ is the solution of the variational equation:

$$
\int_{\Omega} \gamma \nabla w_{j k} \cdot \nabla \varphi d x=\int_{\Omega}\left(\gamma_{0}-\gamma\right) \nabla v_{j k} \cdot \nabla \varphi d x \quad(\varphi \in W)
$$

Since

$$
\sup _{\|\varphi\|_{W} \leq 1}\left|\int_{\Omega}\left(\gamma_{0}-\gamma\right) \nabla\left(v_{j k}-v_{j l}\right) \cdot \nabla \varphi d x\right| \leq\left\|h \gamma_{0}\right\|_{L^{\infty}(D)}\left\|v_{j k}-v_{j l}\right\|_{\bar{H}^{1}(D)} \rightarrow 0
$$

as $k, l \rightarrow \infty$ by $\mathcal{C}_{t} \cap \bar{D}=\phi$ and $v_{j k} \rightarrow v_{j}^{\prime}+H_{j}(\cdot, c(t)) \quad(k \rightarrow \infty)$ in $\bar{H}_{\text {loc }}^{1}\left(\Omega \backslash \mathcal{C}_{t}\right)$, we have from $(1.8)$

$$
\left\|w_{j k}-w_{j l}\right\|_{\bar{H}^{1}(\Omega)} \rightarrow 0 \quad(k, l \rightarrow \infty) .
$$

Hence, there exist limits $w_{j}:=\lim _{k \rightarrow \infty} w_{j k} \in \bar{H}^{1}(\Omega)(j=1,2)$ and they satisfy

$$
\left\{\begin{array}{l}
L_{\gamma} w_{j}=\nabla \cdot\left(\gamma_{0}-\gamma\right) \nabla\left(v_{j}^{\prime}+H_{j}(\cdot, c(t))\right) \text { in } \Omega \\
w_{j}=0 \text { on } \Gamma_{D}, \quad \partial_{L_{\gamma}} w_{j}=0 \text { on } \Gamma_{N} .
\end{array}\right.
$$

Therefore, by Theorem D.1 in Appendix D, we have

$$
\begin{aligned}
\int_{D} h \gamma^{-1}\left(\gamma_{0} \nabla V_{1}\right) \cdot\left(\gamma_{0} \nabla V_{1}\right) d x-\int_{\Omega} F w_{1} d x+\left\langle g, w_{1}\right\rangle_{2} \leq I_{1}(t, \mathcal{C}) \\
\leq \int_{D} h \gamma_{0} \nabla V_{1} \cdot \nabla V_{1} d x-\int_{\Omega} F w_{1} d x+\left\langle g, w_{1}\right\rangle_{2}
\end{aligned}
$$

and

$$
\begin{aligned}
\int_{D} h \gamma^{-1}\left(\gamma_{0} \nabla V_{2}\right) \cdot\left(\gamma_{0} \nabla V_{2}\right) d x- & \int_{\Omega} F w_{2} d x+\left\langle\partial_{L_{\gamma_{0}}} w_{2}, f\right\rangle_{1} \leq I_{2}(t, \mathcal{C}) \\
& \leq \int_{D} h \gamma_{0} \nabla V_{2} \cdot \nabla V_{2} d x-\int_{\Omega} F w_{2} d x-\left\langle\partial_{L_{\gamma_{0}}} w_{2}, f\right\rangle_{1},
\end{aligned}
$$

where $V_{j}(j=1,2)$ are defined by $V_{j}:=v_{j}^{\prime}+H_{j}(\cdot, c(t))(j=1,2)$. 


\section{BEHAVIOR OF INDICATOR FUNCTIONS}

In this Section we analyze the behavior of the indicator functions $I_{j}(t, \mathcal{C})$ as $t \uparrow T(\mathcal{C}, D)$ when $\mathcal{C} \cap \bar{D} \neq \phi$. Hereafter, constants $C, C^{\prime}>0$ which will appear in the estimates are general constants.

Let $\mathcal{C} \cap \bar{D} \neq \phi$ and $0<t<1$ satisfy $\mathcal{C}_{t} \cap \bar{D}=\phi$.

LEMMA 4.1 There exists a constant $M>0$ independent of $t$ such that

$$
\left\|w_{j}\right\|_{L^{2}(\Omega)} \leq M \quad(j=1,2) \quad \text { as } t \uparrow T(\mathcal{C}, D) .
$$

Proof For simplicity, put $t_{0}:=T(\mathcal{C}, D)$ and $a=c\left(t_{0}\right)$. For $0<t<t_{0}$, let $z_{j} \in \bar{H}^{1}(\Omega)$ $(j=1,2)$ be the solutions to

$$
\left\{\begin{array}{l}
L_{\gamma} z_{j}=w_{j} \\
z_{j}=0 \text { on } \Gamma
\end{array}\right.
$$

Then, by (3.7) and $z_{j} \in W$, we have

$$
\int_{\Omega}\left|w_{j}\right|^{2} d x=-\int_{\Omega} \gamma \nabla w_{j} \cdot \nabla z_{j} d x=\int_{D} h \gamma_{0} \nabla V_{j} \cdot \nabla z_{j} d x
$$

It is easy to see that

$$
\left|\int_{D} h \gamma_{0} \nabla v_{j}^{\prime} \cdot \nabla z_{j} d x\right|,\left|\int_{D \backslash B_{\delta}(a)} h \gamma_{0} \nabla H(x, c(t)) \cdot \nabla z_{j} d x\right| \leq C\left\|w_{j}\right\|_{L^{2}(\Omega)}
$$

as $t \uparrow t_{0}$ for some constant $C>0$ independent of $t$. Since $h \in C^{0, \alpha}$ near $\partial D$ with $0<\alpha<1$ for $n=2$ and $\frac{1}{2}<\alpha<1$ for $n=3$, there exists $\eta>0$ such that

$$
\left|\left(h \gamma_{0}\right)(x)-\left(h \gamma_{0}\right)(c(t))\right| \leq K|x-c(t)|^{\alpha} \quad\left(0<t_{0}-t \leq \eta, \quad x \in B_{\delta}(a)\right)
$$

for some constant $K>0$ independent of $t$ and $x$. Hence,

$$
\begin{aligned}
\mid \int_{D \cap B_{\delta}(a)}\left(\left(h \gamma_{0}\right)(x)-\left(h \gamma_{0}\right)(c(t))\right) \nabla H(x, c(t)) & \cdot \beta \nabla z_{j}(x) d x \mid \\
& \leq C K \int_{D \cap B_{\delta}(a)}|x-c(t)|^{\beta}\left|\nabla z_{j}(x)\right| d x
\end{aligned}
$$

where $\beta=-1+\alpha$ for $n=2,-2+\alpha$ for $n=3$ and $C>0$ is some constant independent of $t$. By $0<\alpha<1$ for $n=2$ and $\frac{1}{2}<\alpha<1$ for $n=3$, there exists a constant $C>0$ independent of $t$ such that

$$
\int_{D \cap B_{\delta}(a)}|x-c(t)|^{2 \beta} d x \leq C \quad\left(0<t-t_{0} \leq \eta\right) .
$$


Hence, we have from (4.6) and (4.7),

$$
\begin{aligned}
& \left|\int_{D \cap B_{\delta}(a)}\left(\left(h \gamma_{0}\right)(x)-h \gamma_{0}(c(t))\right) \nabla H(x, c(t)) \cdot \beta \nabla z_{j}(x) d x\right| \leq C\left\|w_{j}\right\|_{L^{2}(\Omega)} \\
& \left(0<t-t_{0} \leq \eta\right)
\end{aligned}
$$

for some constant $C>0$ independent of $t$. Moreover, by (2.3)

$$
\begin{array}{r}
\left|\int_{D \cap B_{\delta}(a)}\left(h \gamma_{0}\right)(c(t)) \nabla(H(x, c(t))-G(x-c(t))) \cdot \nabla z_{j}(x) d x\right| \leq C\left\|w_{j}\right\|_{L^{2}(\Omega)} \\
\left(0<t-t_{0} \leq \eta\right)
\end{array}
$$

for some constant $C>0$ independent of $t$. Therefore, the proof is complete if we show

$$
\left|\int_{D \cap B_{\delta}(a)} \gamma_{0}(c(t)) \nabla G(x-c(t)) \cdot \nabla z_{j}(x) d x\right| \leq C\left\|w_{j}\right\|_{L^{2}(\Omega)} \quad\left(0<t-t_{0} \leq \eta\right)
$$

for some constant $C>0$ independent of $t$.

Since $\nabla \cdot \gamma_{0}(c(t)) \nabla G(x-c(t))=0$ in $D \cap B_{\delta}(a)$,

$$
\begin{gathered}
\int_{D \cap B_{\delta}(a)} \gamma_{0}(c(t)) \nabla G(x-c(t)) \cdot \nabla z_{j}(x) d x=\int_{\partial\left(D \cap B_{\delta}(a)\right)}\left(\gamma_{0}(c(t)) \nabla G(x,-c(t)) \cdot \nu\right) z_{j} d \sigma \\
=\int_{\partial\left(D \cap B_{\delta}(a)\right)}\left(\gamma_{0}(c(t)) \nabla G(x,-c(t)) \cdot \nu\right)\left(z_{j}-z_{j}(c(t))\right) d \sigma
\end{gathered}
$$

By Theorem B.1 in Appendix B, there exist some $\sigma(0<\sigma<1)$ and a constant $C>0$ depending only on $n, C_{1}$ and $\|\gamma\|_{L^{\infty}(\Omega)}$ such that

$$
\left|z_{j}(x)-z_{j}(c(t))\right| \leq C|x-c(t)|^{\sigma}\left\|w_{j}\right\|_{L^{2}(\Omega)} \quad\left(x \in B_{\delta}(a), \quad 0<t-t_{0} \leq \eta\right) .
$$

Hence,

$$
\begin{aligned}
\left|\int_{D \cap B_{\delta}(a)} \gamma_{0}(c(t)) \nabla G(x-c(t)) \cdot \nabla z_{j}(x) d x\right| & \leq C \int_{\partial\left(D \cap B_{\delta}(a)\right)}|x-c(t)|^{-n+1+\sigma} d \sigma\|w\|_{L^{2}(\Omega)} \\
& \leq C^{\prime}\|w\|_{L^{2}(\Omega)} \quad\left(0<t-t_{0} \leq \eta\right)
\end{aligned}
$$

for some constants $C, C^{\prime}>0$ independent of $t$. 
Let $\alpha \in C_{0}^{\infty}(\Omega)$ satisfy $\alpha=1$ in an open neighborhood of $\bar{D}$ and $\widetilde{w}_{j}:=w_{j}-\alpha w_{j}(j=1,2)$. From $(3.7)$ and $\operatorname{supp}\left(\gamma-\gamma_{0}\right) \subset \bar{D}$, we have

$$
(1-\alpha) L_{\gamma} w_{j}=0 \quad \text { in } \Omega
$$

and

$$
L_{\gamma} \alpha=L_{\gamma_{0}} \alpha
$$

Then $\widetilde{w}_{j}$ satisfies

$$
\left\{\begin{array}{l}
L_{\gamma} \widetilde{w}_{j}=F_{j} \text { in } \Omega \\
\widetilde{w}_{j}=0 \text { on } \Gamma_{D}, \partial_{L_{\gamma}} \widetilde{w}_{j}=0 \text { on } \Gamma_{N},
\end{array}\right.
$$

where $F_{j}:=-\left(L_{\gamma} \alpha\right) w_{j}-2 \gamma \nabla \alpha \cdot \nabla w_{j}$ satisfies $\operatorname{supp} F_{j} \subset \Omega$ and $\left\|F_{j}\right\|_{W^{*}}$ is uniformly bounded for any $t\left(0<t-t_{0} \leq \eta\right)$. Therefore, by the continuity of the trace and $\partial_{L_{\gamma_{0}}} w_{2}=\partial_{L_{\gamma}} \widetilde{w}_{2}$,

$$
\left\|w_{1}\right\|_{\bar{H}^{\frac{1}{2}}\left(\Gamma_{N}\right)}+\left\|\partial_{L_{\gamma_{0}}} w_{2}\right\|_{\dot{H}^{-\frac{1}{2}}\left(\overline{\Gamma_{D}}\right)} \leq M \quad \text { as } t \uparrow T(\mathcal{C}, D)
$$

for another constant $M>0$ independent of $t$.

Now it is easy to see that the dominant parts of $\int_{D} h \gamma^{-1}\left(\gamma_{0} \nabla V_{j}\right) \cdot\left(\gamma_{0} \nabla V_{j}\right) d x$ and $\int_{D} h \nabla V_{j} \cdot \nabla V_{j} d x$ are $\int_{D \cap B_{\delta}(a)} h \gamma^{-1}\left(\gamma_{0} \nabla G_{j}(x-c(t))\right) \cdot\left(\gamma_{0} \nabla G_{j}(x-c(t))\right) d x$ and $\int_{D \cap B_{\delta}(a)} h \gamma_{0} \nabla G_{j}(x-c(t)) \cdot \nabla G_{j}(x-c(t)) d x$ which are positive or negative according to (1.3) or (1.4) and blow up as $t \uparrow t_{0}$. Therefore, by (3.8), (3.9), (4.1) and (4.17),

$$
\left|I_{j}(t, \mathcal{C})\right| \rightarrow \infty \quad\left(t \uparrow t_{0}\right) .
$$

Finally, (2.13) can be proven by the standard argument given in [3], so we omit its proof.

\section{APPENDIX}

\section{A BOUNDARY VALUE PROBLEM FOR FORWARD PROBLEM}

In this Appendix we give the proof of the well-posedness of the boundary value problem (1.6).

THEOREM A.1 If $\gamma \in L^{\infty}(\Omega)$ satisfies $\gamma \geq \delta$ in $\Omega$, there exists a unique solution of (1.6). Moreover u satisfies (1.8). 
Proof For $f \in \bar{H}^{\frac{1}{2}}\left(\Gamma_{D}\right)$, there exists $\tilde{f} \in \bar{H}^{\frac{1}{2}}(\Gamma)$ which is the extension of $f$.

Let $\widetilde{u} \in \bar{H}^{1}(\Omega)$ be the solution to

$$
\left\{\begin{array}{l}
L_{\gamma} \widetilde{u}=0 \text { in } \Omega \\
\widetilde{u}=\widetilde{f} \text { on } \Gamma .
\end{array}\right.
$$

Then, it is well known that

$$
\|\widetilde{u}\|_{\bar{H}^{1}(\Omega)} \leq C\|\widetilde{f}\|_{\bar{H}^{\frac{1}{2}}(\Gamma)} \leq C^{\prime}\|f\|_{\bar{H}^{\frac{1}{2}}\left(\Gamma_{D}\right)}
$$

and

$$
\left\|\partial_{L_{\gamma}} \widetilde{u}\right\|_{\bar{H}^{-\frac{1}{2}}(\Gamma)} \leq C\|\widetilde{u}\|_{\bar{H}^{1}(\Omega)} \leq C^{\prime}\|f\|_{\bar{H}^{\frac{1}{2}}\left(\Gamma_{D}\right)}
$$

for some constant $C, C^{\prime}>0$ independent of $f$.

Put $v:=u-\widetilde{u} \in W$. This $v$ has to satisfy

$$
\left\{\begin{array}{l}
L_{\gamma} v=F \quad \text { in } \Omega \\
v=0 \text { on } \Gamma_{D}, \quad \partial_{L_{\gamma}} v=\widetilde{g} \quad \text { on } \Gamma_{N}
\end{array}\right.
$$

where $\widetilde{g}=g-\partial_{L_{\gamma}} \widetilde{u}$. Define

$$
\langle G, w\rangle:=\langle F, w\rangle-\int_{\Gamma_{N}} \tilde{g} w d \Gamma
$$

and

$$
B[v, w]:=\int_{\Omega} \gamma \nabla v \cdot \nabla w d x
$$

for any $v, w \in W$.

By the Schwarz inequality,

$$
|B[v, w]| \leq \int_{\Omega}|\gamma||\nabla v||\nabla w| d x \leq M\|v\|_{\bar{H}^{1}(\Omega)}\|w\|_{\bar{H}^{1}(\Omega)} .
$$

By the Poincaré inequality

$$
B[v, v] \geq \int_{\Omega} \gamma|\nabla v|^{2} d x \geq \delta\|\nabla v\|_{L^{2}(\Omega)} \geq \delta^{\prime}\|v\|_{\bar{H}^{1}(\Omega)}
$$

for some constant $\delta^{\prime}>0$ independent of $v, w$.

Now we remind the Lax-Milgram theorem. 
THEOREM A.2 (Lax-Milgram) Let $X$ be a real Hilbert space and $B: X \times X \rightarrow \boldsymbol{R}$ be a bilinear map satisfying

$$
|B[x, y]| \leq \gamma\|x\|\|y\|
$$

and

$$
B[x, x] \geq \delta\|x\|^{2},
$$

then there exists a unique bounded linear bijective operator $S: X \rightarrow X$ such that

$$
(x, y)=B[S x, y] \quad \text { with }\|S\| \leq \delta^{-1},\left\|S^{-1}\right\| \leq \gamma .
$$

By applying Theorem A.2, there exists a unique bounded linear bijective operator $S: W \rightarrow W$ such that

$$
\left(S^{-1} v, w\right)=B[v, w] \quad(v, w \in W) \quad \text { with }\|S\| \leq\left(\delta^{\prime}\right)^{-1}, \quad\left\|S^{-1}\right\| \leq M .
$$

As immediate estimates, we have

$$
|\langle F, w\rangle| \leq\|F\|_{W^{*}}\|w\|_{W}
$$

and

$$
\begin{aligned}
\left|\int_{\Gamma_{N}} \widetilde{g} w d \Gamma\right| & \leq C\left(\|g\|_{\bar{H}^{-\frac{1}{2}}\left(\Gamma_{N}\right)}+\left\|\partial_{L_{\gamma}} \widetilde{u}\right\|_{\bar{H}^{-\frac{1}{2}}(\Gamma)}\right)\|w\|_{W} \\
& \leq C^{\prime}\left(\|g\|_{\bar{H}^{-\frac{1}{2}}\left(\Gamma_{N}\right)}+\|f\|_{\bar{H}^{\frac{1}{2}\left(\Gamma_{D}\right)}}\right)\|w\|_{W}
\end{aligned}
$$

for some constants $C, C^{\prime}>0$ independent of $f, g, F$. Hence, by (A.13) and (A.14),

$$
|\langle G, w\rangle| \leq C\left(\|f\|_{\bar{H}^{\frac{1}{2}}\left(\Gamma_{D}\right)}+\|g\|_{\bar{H}^{-\frac{1}{2}\left(\Gamma_{N}\right)}}+\|F\|_{W^{*}}\right)\|w\|_{W} .
$$

By the Riesz representation theorem, the exists a unique $\widetilde{v} \in W$ such that

$$
\langle G, w\rangle=-(\widetilde{v}, w), \quad\|\widetilde{v}\|_{W}=\|G\|_{W^{*}}
$$

Let $v \in W$ be $\widetilde{v}=S^{-1} v$.

Then, by (A.12),

$$
B[v, w]+\langle G, w\rangle=0 \quad(w \in W) .
$$

Therefore, $v$ is the solution to (A.4). By the definition of $v$,

$$
\begin{aligned}
\|u\|_{\bar{H}^{1}(\Omega)} & \leq\|v\|_{\bar{H}^{1}(\Omega)}+\|\widetilde{u}\|_{\bar{H}^{1}(\Omega)} \leq C\left(\|\widetilde{v}\|_{\bar{H}^{1}(\Omega)}+\|\widetilde{f}\|_{\bar{H}^{\frac{1}{2}}(\Gamma)}\right) \\
& \leq C^{\prime}\left(\|G\|_{W^{*}}+\|f\|_{\bar{H}^{\frac{1}{2}}\left(\Gamma_{D}\right)}\right) \leq C^{\prime \prime}\left(\|f\|_{\bar{H}^{\frac{1}{2}\left(\Gamma_{D}\right)}}+\|g\|_{\bar{H}^{-\frac{1}{2}}\left(\Gamma_{N}\right)}+\|F\|_{W^{*}}\right)
\end{aligned}
$$

for some constants $C, C^{\prime}, C^{\prime \prime}>0$ independent of $f, g, F$. 


\section{B DE GIORGI-NASH-MOSER THEOREM}

We provide the De Giorgi-Nash-Moser theorem for the readers' convenience. For its proof see [1].

THEOREM B.1 Let $\Omega$ be as given in Section 1 and $\gamma=\left(\gamma_{i j}(x)\right) \in L^{\infty}(\Omega)$ be a symmetric matrix satisfying (1.2). For a given $f \in L^{q}(\Omega)$ for some $q>\frac{n}{2}$, let $u \in \bar{H}^{1}(\Omega)$ satisfy

$$
\int_{\Omega} \gamma_{i j} \partial_{i} u \partial_{j} \varphi d x=\int_{\Omega} f \varphi d x \quad\left(\varphi \in \dot{H}^{1}(\bar{\Omega})\right)
$$

Then, $u \in C^{0, \sigma}(\Omega)$ for some $\sigma(0<\sigma<1)$ depending only $n, q, C_{1}$ and $\|\gamma\|_{L^{\infty}(\Omega)}$. Moreover, for any compact $K \subset \Omega$ and open ball $B_{R}$ with radius $R$ such that $\overline{B_{R}} \subset K$, we have the estimate:

$$
|u(x)-u(y)| \leq C\left(\frac{|x-y|}{R}\right)^{\sigma}\left(R^{-\frac{n}{2}}\|u\|_{L^{2}\left(B_{R}\right)}+R^{2-\frac{n}{q}}\|f\|_{L^{q}\left(B_{R}\right)}\right) \quad\left(x, y \in B_{\frac{R}{2}}\right)
$$

where $B_{\frac{R}{2}}, B_{R}$ are equicentered and $C>0$ is a constant depending only on $n, q, K, C_{1},\|\gamma\|_{L^{\infty}(\Omega)}$.

REMARK B.2 $\Omega$ can be any domain in $\boldsymbol{R}^{n}$ with $n \geq 2 ; n \in \boldsymbol{N}$.

\section{RUNGE'S THEOREM}

In this Appendix two Runge's approximation theorems are given and they are applied to construct the two sequences of approximate functions $\left\{v_{1 k}\right\}$ and $\left\{v_{2 k}\right\}$ given in Section 2 .

Let $\Omega, \Gamma, \Gamma_{D}, \Gamma_{N}, \gamma_{0}$ and $L_{\gamma_{0}}$ be as in Section 1 . Then, we have the first Runge's approximation theorem.

THEOREM C.1 Let $U$ be an open subset of $\Omega$ such that $\bar{U} \subset \Omega$ and $\Omega \backslash \bar{U}$ is connected. Define

$$
\left\{\begin{array}{l}
X=\left\{\left.u\right|_{U} ; u \in \bar{H}^{1}(\widetilde{U}), L_{\gamma_{0}} u=0 \text { in an open neighborhood } \widetilde{U} \text { of } U\right\} \\
Y=\left\{\left.v\right|_{\Omega} ; v \in \bar{H}^{1}(\widetilde{U}), L_{\gamma_{0}} v=0 \text { in } \Omega,\left.\partial_{L_{\gamma_{0}}} v\right|_{\Gamma_{N}}=0, \operatorname{supp}\left(\left.v\right|_{\Gamma_{D}}\right) \subset \Gamma_{0}\right\},
\end{array}\right.
$$

where $\widetilde{U}$ is an open subset of $\Omega$ depending on $u$ such that

$$
\bar{U} \subset \widetilde{U} \subset \overline{\widetilde{U}} \subset \Omega
$$

and $\Gamma_{0}$ is a fixed open subset of $\Gamma_{D}$. Then, $Y$ is dense in $X$ with respect to $\bar{H}^{1}(U)$ topology. 
Proof By the Hahn-Banach theorem, it is enough to prove

$$
f \in \bar{H}^{1}(\widetilde{U})^{*}, f\left(\left.v\right|_{U}\right)=0(v \in Y) \Longrightarrow f\left(\left.u\right|_{U}\right)=0 \quad(u \in X) .
$$

Suppose $f \in \bar{H}^{1}(\widetilde{U})^{*}, f\left(\left.v\right|_{U}\right)=0 \quad(v \in Y)$.

Let $y \in \Gamma_{0}$ and take a small open ball $B$ centered at $y$ and $\Omega_{0}:=\Omega \cup B$. We extend $\gamma_{0} \in C^{0,1}(\bar{\Omega})$ to a neighborhood of $\overline{\Omega_{0}}$ preserving its regularity. Also, let

$$
T:\left\{\Psi \in \bar{H}^{1}(\widetilde{U}) ;\left.\Psi\right|_{\Gamma_{D}}=0\right\} \rightarrow \boldsymbol{R}, \quad T(\Psi)=f\left(\left.\Psi\right|_{U}\right)
$$

$T$ has a bounded linear extension $\widetilde{T} \in \bar{H}^{1}(\Omega)^{*}$. Hence, by the unique solvability to variational problem, there exists $w \in \bar{H}^{1}(\Omega)$ such that $w=0$ in $\Gamma_{D}$ and

$$
-\int_{\Omega} \gamma_{0} \nabla w \cdot \nabla \Psi d x=\widetilde{T}(\Psi) \quad\left(\Psi \in \bar{H}^{1}(\Omega),\left.\Psi\right|_{\Gamma_{D}}=0\right)
$$

Therefore

$$
-\int_{\Omega} \gamma_{0} \nabla w \cdot \nabla \Psi d x=f\left(\left.\Psi\right|_{U}\right) \quad\left(\Psi \in \bar{H}^{1}(\Omega),\left.\Psi\right|_{\Gamma_{D}}=0\right)
$$

Define $\widetilde{w}$ by

$$
\widetilde{w}= \begin{cases}w & \text { in } \Omega \\ 0 & \text { in } \Omega_{0} \backslash \Omega .\end{cases}
$$

Since

$$
\begin{gathered}
\left.w\right|_{\Gamma_{D}}=0, \\
\widetilde{w} \in \bar{H}^{1}(\widetilde{\Omega}) .
\end{gathered}
$$

Now we claim the following.

\section{Claim}

$$
\int_{\Omega_{0}} \gamma_{0} \nabla \widetilde{w} \cdot \nabla \varphi=f\left(\left.\varphi\right|_{U}\right) \quad\left(\varphi \in \dot{H}^{1}(\bar{\Omega})\right)
$$

The proof of this claim will be given later.

From this claim,

$$
L_{\gamma_{0}} \widetilde{w}=0 \text { in } \Omega_{0} \backslash \bar{U}
$$

Note that

$$
\left\{\begin{array}{l}
\widetilde{w}=0 \text { in } \Omega_{0} \backslash \bar{\Omega} \supset \Omega_{0} \backslash \bar{U} \\
\Omega_{0} \backslash \bar{U} \text { is connected. }
\end{array}\right.
$$

Hence, by the weak unique continuation theorem for $L_{\gamma_{0}}$ due to $\gamma_{0} \in C^{0,1}\left(\overline{\Omega_{0}}\right)$, we have

$$
\widetilde{w}=0 \text { in } \Omega_{0} \backslash \bar{U}
$$


Therefore

$$
w=0 \text { in } \Omega \backslash \bar{U}
$$

Now let $v \in X$. Then, for some $\widetilde{U}$ which is an open neighborhood of $\bar{U}$, there exists $u \in \bar{H}^{1}(\widetilde{U})$ such that

$$
L_{\gamma_{0}} u=0 \text { in } \widetilde{U},\left.u\right|_{U}=v .
$$

By taking a cut off function, for some $\widetilde{\widetilde{U}} \subset \widetilde{U}$ which is an open neighborhood of $\bar{U}$, there exists $\widetilde{u} \in \dot{H}^{1}(\bar{\Omega})$ such that

$$
\left.\widetilde{u}\right|_{\widetilde{\widetilde{U}}}=\left.u\right|_{\widetilde{\widetilde{U}}}
$$

Hence, by reminding (C.6) and (C.14), w $\dot{H}^{1}(\widetilde{\widetilde{\widetilde{U}}})$ and $L_{\gamma_{0}} u=0$ in $\widetilde{\widetilde{U}}$,

$$
\begin{aligned}
f(v)=f\left(\left.u\right|_{U}\right)=f\left(\left.\widetilde{u}\right|_{U}\right) & =\int_{\Omega} \gamma_{0} \nabla w \cdot \nabla \widetilde{u} d x \\
& =\int_{\widetilde{\widetilde{U}}} \gamma_{0} \nabla w \cdot \nabla \widetilde{u} d x=\int_{\widetilde{\widetilde{U}}} \gamma_{0} \nabla w \cdot \nabla u d x=0 .
\end{aligned}
$$

Finally, we prove the claim. For any $\varphi \in \dot{H}^{1}\left(\bar{\Omega}_{0}\right)$,

$$
\int_{\Omega_{0}} \gamma_{0} \nabla \widetilde{w} \cdot \nabla u d x=\int_{\Omega_{0} \backslash \bar{\Omega}} \gamma_{0} \nabla \widetilde{w} \cdot \nabla u d x+\int_{\Omega} \gamma_{0} \nabla \widetilde{w} \cdot \nabla u d x=\int_{\Omega} \gamma_{0} \nabla \widetilde{w} \cdot \nabla u d x
$$

Let $v \in \bar{H}^{1}(\Omega)$ be the solution to

$$
\left\{\begin{array}{l}
L_{\gamma_{0}} v=0 \text { in } \Omega \\
\partial_{L_{\gamma_{0}}} v=0 \text { in } \Gamma_{N}, v=\varphi \text { in } \Gamma_{D}
\end{array}\right.
$$

Clearly,

$$
v-\varphi \in \bar{H}^{1}(\Omega),\left.(v-\varphi)\right|_{\Gamma_{D}}=0
$$

By (C.6),

$$
-\int_{\Omega} \gamma_{0} \nabla w \cdot \nabla(v-\varphi) d x=f\left(\left.v\right|_{U}-\left.\varphi\right|_{U}\right)
$$

Here note that $\left.v\right|_{U} \in Y$ by $\operatorname{supp}\left(\left.v\right|_{\Gamma_{D}}\right) \subset \Gamma_{0}$,

$$
f\left(\left.v\right|_{U}\right)=0
$$

On the other hand, remind that

$$
w \in \bar{H}^{1}(\Omega),\left.w\right|_{\Gamma_{D}}=0 \text { and } L_{\gamma_{0}} v=0 \text { in } \Omega,\left.\partial_{\gamma_{0}} v\right|_{\Gamma_{N}}=0,\left.v\right|_{\Gamma_{D}}=\varphi .
$$


By the definition of weak solution,

$$
\int_{\Omega} \gamma_{0} \nabla w \cdot \nabla v d x=0
$$

By (C.7), (C.19), (C.20) and (C.22),

$$
-\int_{\Omega} \gamma_{0} \nabla \widetilde{w} \cdot \nabla \varphi d x=f\left(\left.\varphi\right|_{U}\right)
$$

Likewise the proof given in [3] we have the second Runge's approximation theorem.

THEOREM C.2 Let $U$ be an open subset of $\Omega$ such that $\bar{U} \subset \Omega$ and $\Omega \backslash \bar{U}$ is connected. Define the two spaces $X, Y$ of functions by

$$
\left\{\begin{array}{l}
X:=\left\{\left.u\right|_{U} ; u \in \bar{H}^{1}(\widetilde{U}), L_{\gamma_{0}} \widetilde{u}=0 \quad \text { in } \widetilde{U}\right\}, \\
Y:=\left\{\left.v\right|_{U} ; v \in \bar{H}^{1}(\Omega), L_{\gamma_{0}} v=0 \quad \text { in } \Omega, \operatorname{supp}\left(\left.v\right|_{\Gamma}\right) \subset \Gamma_{0}\right\},
\end{array}\right.
$$

where $\widetilde{U}$ is an open subset of $\Omega$ depending on u such that $\bar{U} \subset \widetilde{U} \subset \overline{\widetilde{U}} \subset \Omega$ and $\Gamma_{0}$ is a fixed open subset of $\Gamma_{N}$. Then, $Y$ is dense in $X$ with respect to $\bar{H}^{1}(U)$ norm.

Next we construct $\left\{v_{j k}\right\} \quad(j=1,2)$. By Theorems C.1, C.2, there exist $\left\{v_{1 k}^{\prime \prime}\right\},\left\{v_{2 k}^{\prime \prime}\right\} \subset \bar{H}^{1}(\Omega)$ such that $v_{j k}^{\prime \prime} \rightarrow H(\cdot, c(t))$ in $\bar{H}_{\text {loc }}^{1}\left(\Omega \backslash \mathcal{C}_{t}\right)$ for each $j(j=1,2)$,

$$
\left\{\begin{array}{l}
L_{\gamma_{0}} v_{1 k}^{\prime \prime}=0 \text { in } \Omega \\
\partial_{L_{\gamma_{0}}} v_{2 k}^{\prime \prime}=0 \text { on } \Gamma_{N}, \quad \operatorname{supp}\left(\left.v_{1 k}^{\prime \prime}\right|_{\Gamma}\right) \subset \Gamma_{10}
\end{array}\right.
$$

and

$$
\left\{\begin{array}{l}
L_{\gamma_{0}} v_{2 k}^{\prime \prime}=0 \quad \text { in } \Omega \\
\operatorname{supp}\left(\left.v_{2 k}^{\prime \prime}\right|_{\Gamma}\right) \subset \Gamma_{20}
\end{array}\right.
$$

where $\Gamma_{10} \subset \Gamma_{D}, \Gamma_{20} \subset \Gamma_{N}$ are open subsets.

Then, we only have to define each $\left\{v_{j k}\right\}(j=1,2)$ by

$$
v_{j k}:=v_{j}^{\prime}+v_{j k}^{\prime \prime}
$$

\section{SOME PRELIMINARY ESTIMATES}

In this Appendix we prove some estimates used in Section 3. Let $u \in \bar{H}^{1}(\Omega)$ be the solution to (1.6) and $v \in \bar{H}^{1}(\Omega)$ be the solution to (1.6) with $\gamma=\gamma_{0}$. Then, we have 


\section{THEOREM D.1}

(i)

$$
\left\langle\left(\Lambda_{\gamma}-\Lambda_{\gamma_{0}}\right) f, f\right\rangle_{1} \leq \int_{D} h \gamma_{0} \nabla v \cdot \nabla v d x-\int_{\Omega} F(u-v) d x+\langle g, u-v\rangle_{2}
$$

and

$$
\left\langle\left(\Lambda_{\gamma}-\Lambda_{\gamma_{0}}\right) f, f\right\rangle_{1} \geq \int_{D} h \gamma^{-1}\left(\gamma_{0} \nabla\right) v \cdot\left(\gamma_{0} \nabla\right) v d x-\int_{\Omega} F(u-v) d x+\langle g, u-v\rangle_{2} .
$$

(ii)

$$
\left\langle g,\left(\Pi_{\gamma}-\Pi_{\gamma_{0}}\right) g\right\rangle_{2} \leq \int_{D} h \gamma_{0} \nabla v \cdot \nabla v d x-\int_{\Omega} F(u-v) d x-\left\langle\partial_{L_{\gamma_{0}}}(u-v), f\right\rangle_{1}
$$

and

$$
\left\langle g,\left(\Pi_{\gamma}-\Pi_{\gamma_{0}}\right) g\right\rangle_{2} \geq \int_{D} h \gamma^{-1}\left(\gamma_{0} \nabla\right) v \cdot\left(\gamma_{0} \nabla\right) v d x-\int_{\Omega} F(u-v) d x-\left\langle\partial_{L_{\gamma_{0}}}(u-v), f\right\rangle_{1} .
$$

Proof We use the inequality given in [3]:

$$
\gamma_{0} \nabla(v-u) \cdot \nabla(v-u)+\left(\gamma-\gamma_{0}\right) \nabla u \cdot \nabla u \geq \gamma_{0}^{-1}\left(\gamma-\gamma_{0}\right) \gamma^{-1}\left(\gamma_{0} \nabla v\right) \cdot\left(\gamma_{0} \nabla v\right) .
$$

We first prove (i). Observe that

$$
\begin{aligned}
\int_{\Omega}\left\{\gamma_{0} \nabla(u-v) \cdot \nabla(u-v)+\right. & \left.\left(\gamma-\gamma_{0}\right) \nabla v \cdot \nabla v\right\} d x \\
& =\int_{\Omega}(\gamma \nabla u \cdot \nabla v-2(\gamma \nabla u) \cdot \nabla v) d x+\int_{\Omega} \gamma_{0} \nabla v \cdot \nabla v d x
\end{aligned}
$$

and

$$
\begin{aligned}
\int_{\Omega}\{\gamma \nabla(v-u) \cdot \nabla(v-u)+ & \left.\left(\gamma_{0}-\gamma\right) \nabla u \cdot \nabla u\right\} d x \\
& =\int_{\Omega}\left(\gamma_{0} \nabla v \cdot \nabla v-2\left(\gamma_{0} \nabla v\right) \cdot \nabla u\right) d x+\int_{\Omega} \gamma \nabla u \cdot \nabla u d x .
\end{aligned}
$$

By the definitions of the Dirichlet to Neumann map and the Neumann to Dirichlet map, we have from (D.6),

$$
\begin{aligned}
& \int_{\Omega}\left\{\gamma \nabla(u-v) \cdot \nabla(u-v)+\left(\gamma_{0}-\gamma\right) \nabla v \cdot \nabla v\right\} d x \\
& =\left\{\begin{array}{l}
\left\langle\left(\Lambda_{\gamma_{0}}-\Lambda_{\gamma}\right) f, f\right\rangle_{1}+\int_{\Omega} F(v-u) d x-\langle g, v-u\rangle_{2} \\
\left\langle g,\left(\Pi_{\gamma_{0}}-\Pi_{\gamma}\right) g\right\rangle_{2}+\int_{\Omega} F(v-u) d x+\left\langle\partial_{L_{\gamma_{0}}}(v-u), f\right\rangle_{1},
\end{array}\right.
\end{aligned}
$$


where $d \sigma$ is the line segment for $n=2$ and the surface measure for $n=3$. Also, we have from (D.7),

$$
\begin{aligned}
\int_{\Omega}\left\{\gamma_{0} \nabla(v-u) \cdot \nabla(v-u)+\left(\gamma-\gamma_{0}\right) \nabla u \cdot \nabla u\right\} d x \\
=\left\{\begin{array}{l}
\left\langle\left(\Lambda_{\gamma}-\Lambda_{\gamma_{0}}\right) f, f\right\rangle_{1}+\int_{\Omega} F(u-v) d x-\langle g, u-v\rangle_{2} \\
\left\langle g,\left(\Pi_{\gamma}-\Pi_{\gamma_{0}}\right) g\right\rangle_{2}+\int_{\Omega} F(u-v) d x+\left\langle\partial_{L_{\gamma_{0}}}(u-v), f\right\rangle_{1} .
\end{array}\right.
\end{aligned}
$$

Reminding (1.2), we have (D.1) and (D.3) from (D.8). Also, by (D.5), we have (D.2) and (D.4) from (D.9).

\section{Acknowledgements}

The second author is partially supported by Grant-in-Aid for Scientific Research (C)(2) (No.13640152) of Japan Society for the Promotion of Science and the third author is partially supported by Grant-in-Aid for Scientific Research (B)(2) (No.14340038) of Japan Society for the Promotion of Science.

\section{References}

[1] Q. Han and F. Lin (2000). Elliptic Partial Differential Equations, AMS. Providence.

[2] L. Hörmander (1985). The Analysis of Linear Partial Differential Operators III, Springer. Berlin.

[3] M. Ikehata (2002). Reconstruction of inclusion from boundary measurements, J.Inverse and Ill-Posed Problems, 10, 37- 65

[4] M. Ikehata and G. Nakamura (submitted). Reconstruction procedure for identifying cracks.

[5] M. Ikehata, G. Nakamura and K. Tanuma (1999). Identification of the shape of the inclusion in the anisotoropic elastic body, Applicable Analysis, 72, 17-26

[6] V. Isakov (1988). On uniquness of recovery of a discontinuous conductivity coefficient, Comm. Pure Appl. Math, 41, 865-877 
[7] R. Potthast (2001). Point sources and multipoles in inverese scattering theory, CHAPMAN \& HALL/CRC. London. 